\title{
C-terminal proendothelin-1 (CT-proET-1) is associated with organ failure and predicts mortality in critically ill patients
}

\author{
Lukas Buendgens ${ }^{1 *}$, Eray Yagmur², Jan Bruensing ${ }^{1}$, Ulf Herbers ${ }^{1}$, Christer Baeck', Christian Trautwein',
} Alexander Koch ${ }^{1}$ and Frank Tacke ${ }^{1}$

\begin{abstract}
Background: Endothelin 1 (ET-1) is a strong vasoconstrictor, which is involved in inflammation and reduced tissue perfusion. C-terminal proendothelin-1 (CT-proET-1) is the stable circulating precursor protein of ET-1. We hypothesized that CT-proET-1, reflecting ET-1 activation, is involved in the pathogenesis of critical illness and associated with its prognosis.

Methods: Two hundred seventeen critically ill patients (144 with sepsis, 73 without sepsis) were included prospectively upon admission to the medical intensive care unit (ICU), in comparison to 65 healthy controls. CT-proET-1 serum concentrations were correlated with clinical data and extensive laboratory parameters. Overall survival was followed for up to 3 years.

Results: CT-proET-1 serum levels at admission were significantly increased in critically ill patients compared to controls. CT-proET-1 serum levels showed significant correlations to systemic inflammation as well as multiple markers of organ dysfunction (kidney, liver, heart). Patients with sepsis displayed higher circulating CT-proET-1 than ICU patients with non-septic diseases. CT-proET-1 levels $>74$ pmol/L at ICU admission independently predicted ICU death (adjusted hazard ratio (HR) 2.66, 95\% confidence interval (Cl) 1.30-5.47) and overall mortality during follow-up (adjusted HR 2.19, 95\%-Cl 1.21-3.98).
\end{abstract}

Conclusions: CT-proET-1 serum concentrations at admission are increased in critically ill patients and associated with sepsis, disease severity, organ failure, and mortality.

Keywords: C-terminal proendothelin-1, CT-proET-1, ICU, Prognosis, Sepsis, Biomarker, Critical illness, Endothelin, ET-1

\section{Background}

Endothelial dysfunction plays an important role in critical illness, especially in sepsis. It mediates hemodynamic disturbances based on the vasotonus, contributes to the balance of pro- and anti-inflammation, regulates nutrient supply and cell migration into tissue, and plays a key role in host-pathogen interaction [1]. Besides other mediators such as nitric oxide (NO), endothelin-1 (ET-1) is one of the major endogenous factors controlling vasotonus that is released from activated endothelial cells. It is the most prominent member of the endothelin family. It

\footnotetext{
* Correspondence: Ibuendgens@ukaachen.de

'Department of Medicine III, RWTH-University Hospital Aachen,

Pauwelsstrasse 30, 52074 Aachen, Germany

Full list of author information is available at the end of the article
}

binds to two G-protein-coupled receptors, $\mathrm{ET}_{\mathrm{A}}$ and $\mathrm{ET}_{\mathrm{B}}$. $\mathrm{ET}_{\mathrm{A}}$ promotes potent vasoconstriction and cell growth, whereas $\mathrm{ET}_{\mathrm{B}}$ leads to vasodilation and inhibits cell proliferation [2]. Besides in blood vessels, ET-1-receptors are also found in tissues, e.g., cardiomyocytes and glomerular capillaries [3]. Endothelin release from endothelial cells is known to be stimulated by bacterial endotoxin [4] and various inflammatory cytokines such as TNFalpha [3] or interleukin-6 [5] as well as mechanical factors like reduced shear stress [6].

Consecutively, increased levels of endothelin were found both in animal models of sepsis $[7,8]$ and human patients with sepsis [9-11]. Moreover, the function of many organs (e.g., liver, lung, heart, or kidney) worsens severely after infusion of ET-1 in animal models $[12,13]$. In the past, 
various smaller studies could relate these findings to the clinical outcome of patients and demonstrated a relation between ET-1 and mortality in sepsis or septic shock in adults $[9,14,15]$ and children [16]. ET-1 itself, however, is difficult to measure due to its limited half-life. Consequently, sample sizes of trials investigating ET-1 tend to be relatively small. The precursor peptide C-terminal proendothelin-1 (CT-proET-1) is far more stable and allows a stoichiometric measurement of ET-1 [17]. This facilitates the analysis of larger group of patients as well as the practical use of this potential biomarker in clinical routine. We therefore investigated CT-proET-1 in a large cohort of 217 consecutively enrolled critically ill patients, including 144 subjects with sepsis, in order to identify associations between CT-proET-1 and organ dysfunction, disease severity as well as ICU, and survival during followup in critically ill patients.

\section{Methods}

\section{Study design}

Written informed consent was obtained from the patient, his or her spouse, or the appointed legal guardian. Patients who were expected to have a short $(<3$ days $)$ intensive care treatment (e.g., due to post-interventional observation or intoxication) were excluded [18]. The long-term course of patients was assessed by directly contacting the patient, the patients' relatives, or their primary care physician. We used the third international consensus definitions for sepsis and septic shock (sepsis3) as a post hoc definition for sepsis patients, and all others were classified as non-sepsis patients [19]. For identifying and classifying patients with an acute respiratory distress syndrome (ARDS), we used the Berlin definition of ARDS [20].

Sixty-five healthy blood donors with normal values for blood counts, C-reactive protein, and liver enzymes served as controls. The study protocol was approved by the local ethics committee and conducted in accordance with the ethical standards laid down in the 1964 Declaration of Helsinki (ethics committee of the University Hospital Aachen, RWTH-University, Aachen, Germany, reference number EK 150/06). The current study was part of a larger assessment of biomarkers in critically ill patients, conducted between 2006 and 2014 at our center.

\section{CT-proET-1 measurements}

Blood samples were collected directly upon admission of the patient to the ICU prior to therapeutic interventions at the ICU. After centrifugation at $4{ }^{\circ} \mathrm{C}$ for $10 \mathrm{~min}$, serum aliquots of $1 \mathrm{~mL}$ were frozen immediately at $-80{ }^{\circ} \mathrm{C}$. CT-proET-1 serum concentrations were measured using a commercially available fluorescent immunoassay (BRAHMS GmbH/ThermoFischer Scientific, Henningsdorf, Germany) following the manufacturer's protocol. The scientist performing laboratory measurements was fully blinded to any clinical or other laboratory data of the patients or controls.

\section{Statistical analysis}

Data are displayed as median and range due to the skewed distribution of most of the parameters. Differences between two groups were assessed by MannWhitney $U$ test or chi-squared test. Differences between multiple groups were assessed using the Kruskal-Wallis test. To illustrate differences between subgroups, box plot graphics were used displaying a summary of the median, quartiles, range, and extreme values of the given data. Their whiskers range from the minimum to the maximum value excluding outliers displayed as separate points. An outlier was defined as a value that is smaller than the lower quartile minus 1.5 times the interquartile range or larger than the upper quartile plus 1.5 times the interquartile range. A far-out value was defined as a value that is smaller than the lower quartile minus three times the interquartile range or larger than the upper quartile plus three times the interquartile range [21]. Correlations between variables were assessed with Spearman correlation tests. The Cox regression model was used for univariate and multivariate analysis of risk factors. Kaplan-Meier curves were used to illustrate differences in survival [22]. Differences between the groups regarding survival were assessed with the log-rank test. Receiver operating characteristic (ROC) curve analysis were used to evaluate the value of a predictive marker or a composite score. ROC curves were generated by plotting sensitivity against 1-specificity [23]. Differences between ROC curves were assessed using the method described by DeLong et al. [24]. Statistical analyses were performed with SPSS Version 23 (SPSS, Chicago, IL, USA) and MedCalc Version 16 (MedCalc Software, Ostend, Belgium).

\section{Results \\ CT-proET-1 serum concentrations are increased in critically ill patients and associated with sepsis}

In order to investigate the role of CT-proET-1 in critical illness, we measured serum levels in 217 patients at the time of admission to our medical ICU. In comparison to 65 healthy controls, CT-proET-1 levels were strongly elevated in critically ill patients (median 5.8 vs $65.4 \mathrm{pmol} /$ L, $p<0.001, U$ test; Fig. 1a).

Of all 217 patients, 144 were admitted because of sepsis. The most frequent septic focus was pneumonia $(n=74)$, followed by abdominal $(n=26)$ and urogenital infections $(n=11)$ (detailed data not shown). Non-septic ICU patients were admitted due to cardio-pulmonary diseases $(n$ $=29)$, pancreatitis $(n=12)$, decompensated liver cirrhosis $(n=9)$, and other non-septic diseases $(n=23)$. CT-proET- 

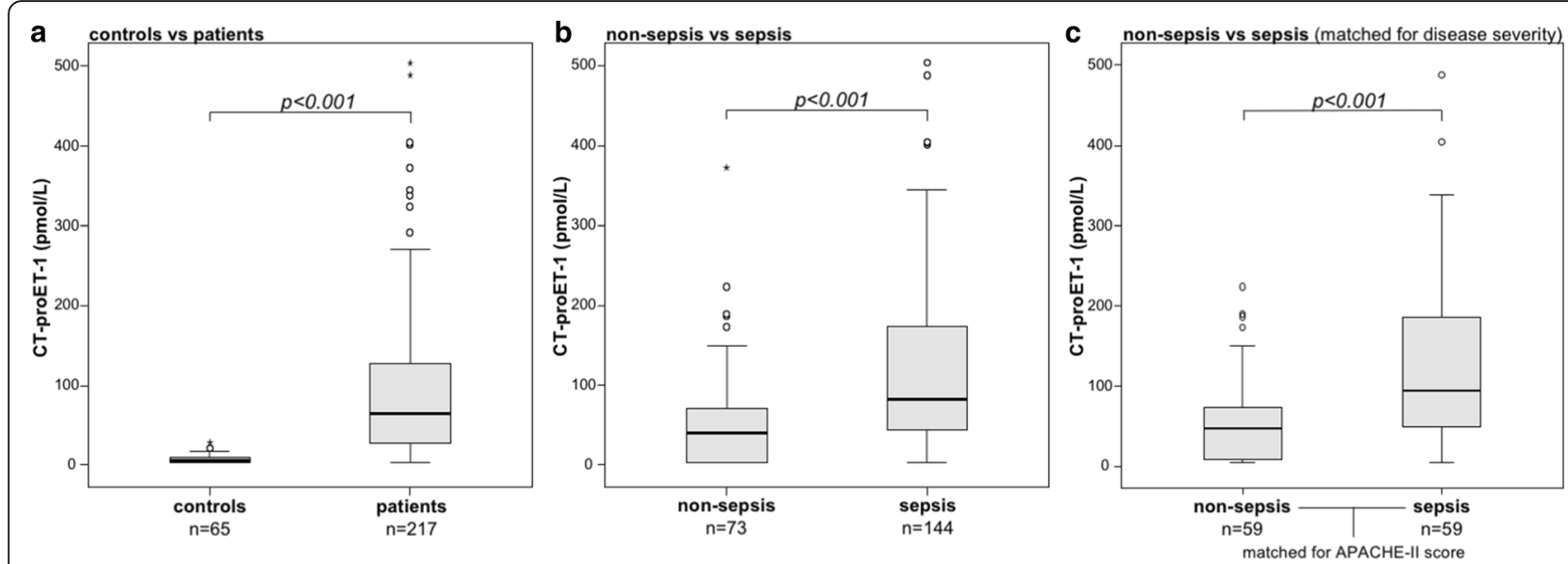

Fig. 1 Serum CT-proET-1 concentrations in critically ill patients and sepsis. a Serum levels of CT-proET-1, at the time of admission to the ICU, were significantly higher in critically ill patients than in healthy controls $(p<0.001 ; U$ test). $\mathbf{b}$ CT-proET-1 levels were significantly higher in patients with sepsis $(p<0.001)$ compared to ICU patients without sepsis. $\mathbf{c}$ When matched 1:1 with non-sepsis patients for APACHE-II score, age, and sex, CT-proET-1 levels were significantly higher in patients with sepsis compared to ICU patients without sepsis $(p<0.001)$

1 levels were significantly higher in patients with sepsis compared to other ICU patients (median 40.9 vs 82.1 pmol/L; $p<0.001$; Fig. 1b, Table 1). Sepsis and non-sepsis patients did not differ in age or sex, but sepsis patients had significantly higher APACHE-II or SOFA scores, increased mortality, longer stay on the ICU, and an increased vasopressor demand (Table 1). In order to exclude that the difference in CT-pro-ET1 was related to the presence of sepsis and not to disease severity, the nonsepsis ICU patients were matched 1:1 with a patient from the sepsis group for APACHE-II score (disease severity), age, and sex. In this subanalysis, CT-pro-ET1 remained indeed higher in the sepsis group ( $p<0.001$; Fig. 1c).

To investigate a possible use of CT-proET-1 in identifying patients with sepsis, we conducted a ROC curve analysis comparing CT-proET-1 to other, established

Table 1 Baseline patient characteristics and CT-proET-1 serum measurements at ICU admission

\begin{tabular}{|c|c|c|c|c|}
\hline Parameter & All patients & Sepsis & Non-sepsis & $p^{a}$ \\
\hline Number & 217 & 144 & 73 & \\
\hline Sex (male/female) & $132 / 85$ & $84 / 60$ & $48 / 25$ & n.s. \\
\hline Age median, (range) [years] & $64(18-90)$ & $65(20-90)$ & $61(18-85)$ & n.s. \\
\hline APACHE-\|l score, median (range) & $18(2-43)$ & $19(4-43)$ & $14(2-33)$ & $<0.001$ \\
\hline SOFA score, median (range) & $9(0-17)$ & $9(2-17)$ & $7(0-17)$ & 0.003 \\
\hline SAPS 2 score, median (range) & $41(0-73)$ & $40(0-73)$ & $41(0-73)$ & 0.193 \\
\hline Mechanical ventilation, $n$ (\%) & $144(69)$ & $98(63)$ & $46(63)$ & n.s. \\
\hline Ventilation time, median (range) [h] & $117(0-3828)$ & $125.5(0-2966)$ & $66(0-2828)$ & n.s. \\
\hline Vasopressor demand, $n$ (\%) & $132(60.8)$ & $99(68.8)$ & $33(45.2)$ & $<0.001$ \\
\hline ICU days, median (range) & $7(1-137)$ & $8.5(1-137)$ & $6(1-45)$ & 0.004 \\
\hline 30-day mortality, n (\%) & $41(18.9)$ & $34(23.6)$ & 7 (9.6) & 0.016 \\
\hline Overall mortality, $n$ (\%) & $86(41.7)$ & $64(46.7)$ & $22(31.9)$ & 0.42 \\
\hline CT-proET-1, median (range) [pmol/L] & $43.8(3-503.6)$ & $82.1(3-503.6)$ & $40.9(3-372.9)$ & $<0.001$ \\
\hline Leucocytes, median (range) [per nL] & $12.9(0.5-208)$ & $13.8(0.5-208)$ & $12.5(1.8-29.6)$ & 0.041 \\
\hline CRP, median (range) $[\mathrm{mg} / \mathrm{L}]$ & $98(5-230)$ & $160.5(5-230)$ & $17(5-230)$ & $<0.001$ \\
\hline Cystatin C, median (range) [mg/L] & $1.48(0.39-8.38)$ & $1.69(0.39-8.38)$ & $1.04(0.56-2.29)$ & $<0.001$ \\
\hline Bilirubin, median (range) [per mg/dL] & $0.7(0.2-20.8)$ & $0.7(0.2-6.8)$ & $0.7(0.2-20.8)$ & n.s. \\
\hline
\end{tabular}

For quantitative variables, median and range (in parenthesis) are given

Abbreviations: CRP C-reactive protein, CT-proET-1 C-terminal proendothelin-1, APACHE Acute Physiology and Chronic Health Evaluation, SAPS 2 Simplified Acute Physiology Score, SOFA Sequential Organ Failure Assessment, n.s. not significant

${ }^{a}$ Significance between sepsis and non-sepsis patients was assessed using the Mann-Whitney $U$ test or chi-squared test 
markers (procalcitonin and C-reactive protein (CRP)). With an AUC of 0.834 (95\%-CI 0.768-0.900), CRP was significantly superior to both PCT ( $p=0.046$; DeLong test) and CT-proET-1 ( $p=0.007$; DeLong test). Interestingly, CT-proET-1 was non-inferior to PCT (AUC 0.704 vs $0.757 ; p=0.24$; DeLong test).

\section{CT-proET-1 levels in critically ill patients correlate with} clinical disease severity scores and organ dysfunction

Based on the pathogenic role of endothelin-1 for vasoconstriction and impaired tissue perfusion [25], we hypothesized that increased CT-proET-1 might be associated with organ dysfunction in ICU patients. Strikingly, CT-proET-1 levels were strongly associated with markers of renal dysfunction (e.g., creatinine, $r=0.500, p<0.001$; cystatin $\mathrm{C}, r$ $=0.624, p<0.001$ ), cholestasis (e.g., bilirubin, $r=0.148, p$ $=0.031$ ), impaired hepatic synthesis (e.g., albumin, $r=$ $-0.321, p=0.001$; pseudocholinesterase, $r=-0.438, p<$ 0.001 ; prothrombin time, $r=-0.220, p=0.001$ ) and cardiac failure (e.g., brain natriuretic peptide, $r=0.505, p<$ 0.001). Likewise, CT-proET-1 levels correlated with markers of general inflammation (e.g., C-reactive protein, $r=0.416, p<0.001$, Table 2).

Interestingly, patients with manifest organ failure had significantly elevated CT-proET-1 levels. This was observed for patients with renal failure (defined as a cystatin C-based glomerular filtration rate below $50 \mathrm{~mL} / \mathrm{min}$, Fig. 2a), liver failure (defined as prothrombin time $<50 \%$, Fig. 2b), or heart failure (defined as a NTproBNP $>1000$ pg/ml; Fig. 2c). As prior studies reported elevated ET-1 in patients with ARDS compared to controls [26], we further assessed CT-proET-1 serum levels in regard to the degree of an ARDS. However, CT-proET-1 did not differ between ICU patients without ARDS $(n=22)$ and with mild $(n=29)$, moderate $(n=27)$, or severe $(n=$ 13) ARDS at the time of admission (Fig. 2d).

Additionally, CT-proET-1 levels were associated with severity of critical illness. Patients with higher APACHE-II (above 18) and SAPS 2 (above the median of the cohort) scores showed significantly increased serum levels of CTproET-1 (Fig. 3a, b). CT-proET-1 also positively correlated with these disease severity scores (APACHE-II, $r=0.239$, $p=0.001$; SAPS $2, r=0.400, p<0.001$, Table 2). CTproET1 did not correlate with the SOFA score, neither for all nor for sepsis patients (detailed data not shown).

\section{CT-proET-1 at admission is an independent predictor of ICU mortality}

As CT-proET-1 levels correlate with organ dysfunction and disease severity, we hypothesized that CT-proET-1 serum concentrations at the time of ICU admission might be associated with mortality in critically ill patients. Overall, $n=41(18.9 \%)$ of the patients died at the ICU, while $n$ $=86(39.6 \%)$ died overall including the follow-up time (of
Table 2 Correlations of CT-proET-1 with clinical scores and other laboratory markers

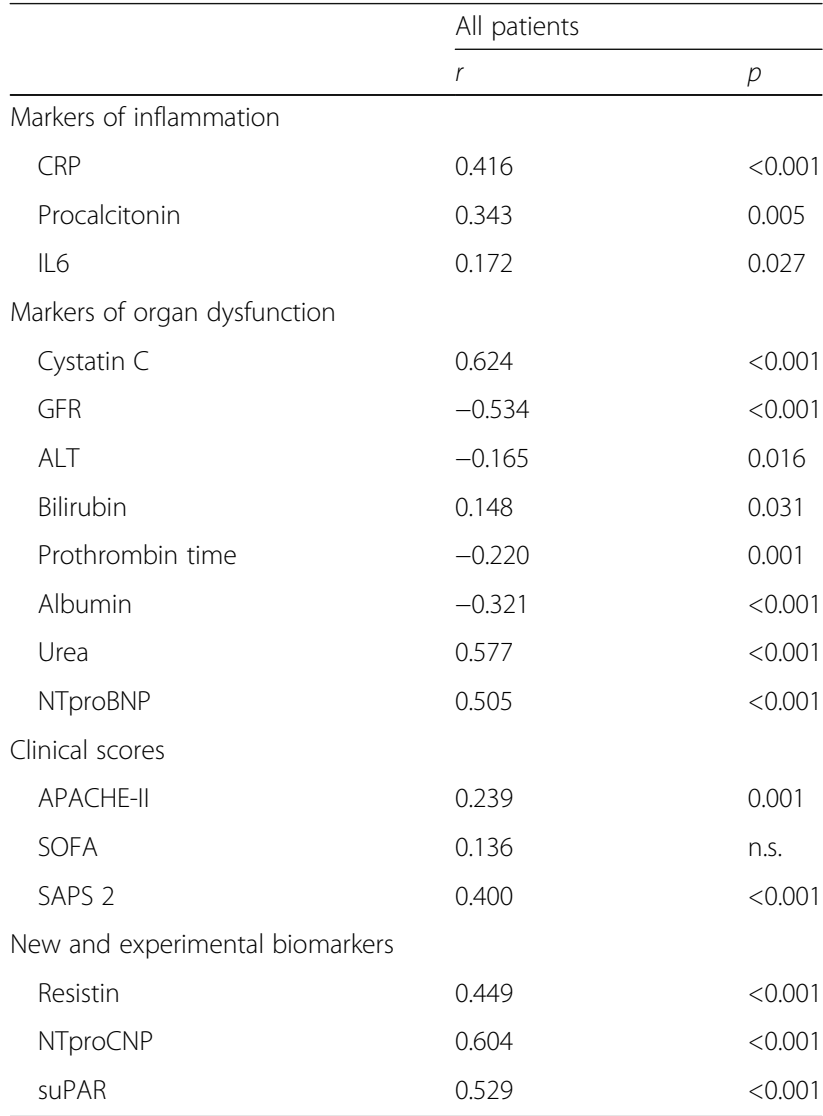

Abbreviations: ALT alanine aminotransferase, APACHE Acute Physiology and Chronic Health Evaluation, ALT alanine aminotransferase, CRP C-reactive protein, GFR glomerular filtration rate, IL6 interleukin 6, NTproBNP amino-terminal propeptide of brain natriuretic peptide, NTproCNP, amino-terminal propeptide of C-type natriuretic peptide, SAPS Simplified Acute Physiology Score, SOFA sequential organ failure assessment, suPAR soluble urokinase plasminogen activator receptor, n.s. not significant

up to 3 years). Remarkably, patients that died at the ICU showed significant higher serum levels of CT-proET-1 at ICU admission than survivors (median 88.3 vs $59.2 \mathrm{pmol} /$ L; $p=0.029$; Fig. 4a).

By Cox regression analysis, CT-proET-1 levels were found to predict ICU mortality $(p=0.047)$. We used the Youden index (28) to find the best cut-off value regarding sensitivity and specificity. Based on the coordinates of the ROC curve, a CT-proET-1 cut-off value of $74 \mathrm{pmol} / \mathrm{L}$ showed the best ratio of sensitivity and specificity in predicting ICU mortality. Interestingly, this value is higher than the measurements of our healthy controls and represents the 99th percentile of a healthy population. KaplanMeier survival curve analysis confirmed that high CTproET-1 levels were strongly associated with 30-day mortality (Fig. 4b; $p=0.002$ ). As CT-proET-1 correlates with markers of organ failure, excretion and inflammation, we next tested if CT-proET-1 serum levels can independently predict survival. We performed uni- and multivariate Cox 


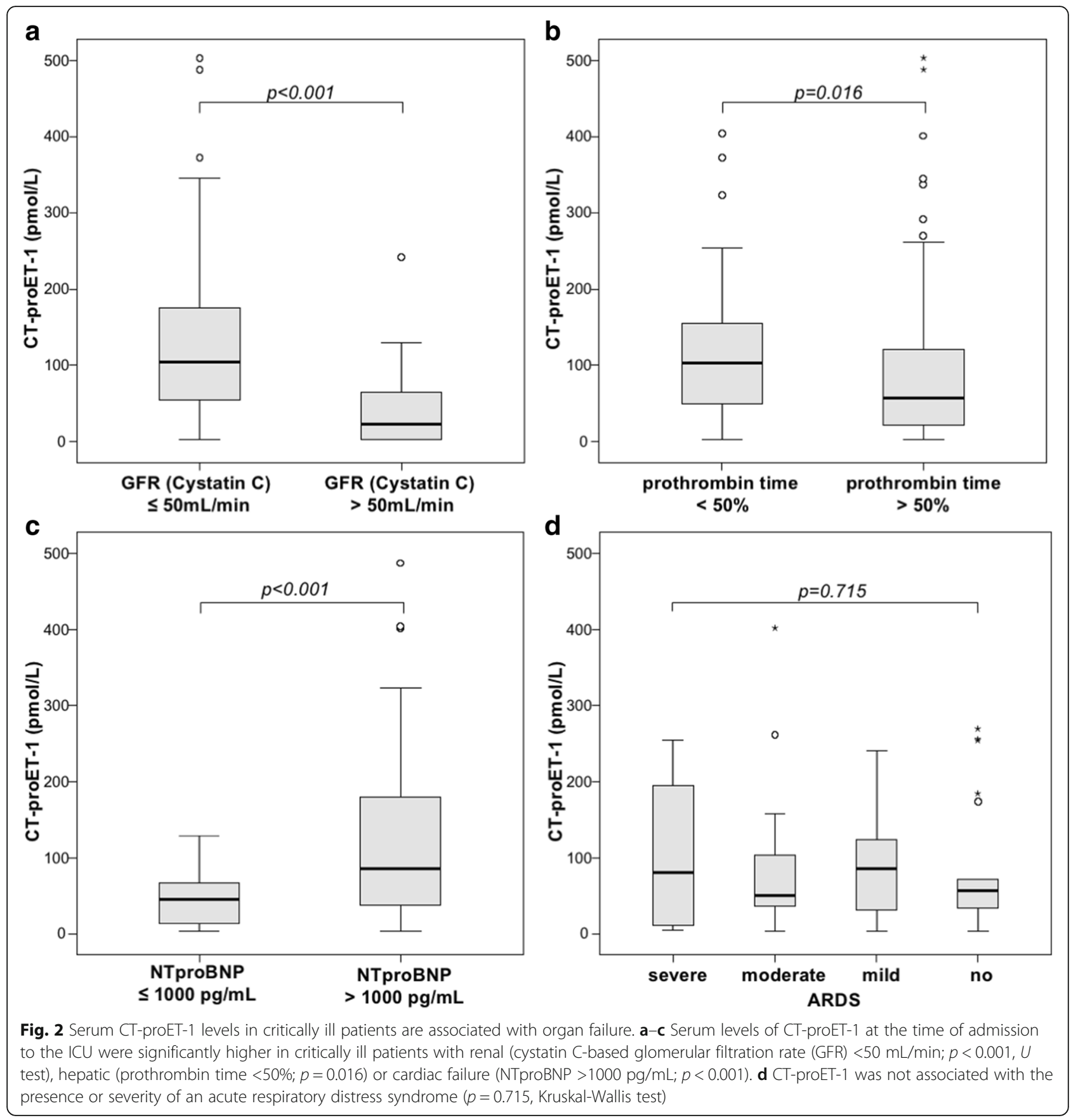

regression analysis including age, markers of inflammation (i.e., CRP), renal (i.e., creatinine), circulatory (i.e., lactate), and hepatic dysfunction (i.e., bilirubin, prothrombin time). Here, high CT-proET-1 (>74 pmol/L) was an independent predictor of ICU mortality in critically ill patients (adjusted hazard ratio (HR) 2.66 (95\% CI 1.30-5.47), Table 3).

High levels of CT-proET-1 at ICU admission are associated with overall survival

Given its association with short-term mortality, we examined if the level of CT-proET-1 at admission to the
ICU was also related to long-term outcome. We found that CT-proET-1 levels were significantly higher in patients that died during the follow-up period compared to the overall surviving patients (median $53.7 \mathrm{vs} 89.9 \mathrm{pmol} /$ L; $p=0.003$; Fig. 5a).

Again, Kaplan-Meier analysis showed a good discrimination in terms of long-term survival between the groups with high and low CT-proET-1 values (Fig. 5b, $p<0.001$ ).

In addition, we conducted uni- and multivariate Cox regression analyses including age, markers of inflammation (i.e., CRP) and renal (i.e., creatinine), circulatory (i.e., lactate), 

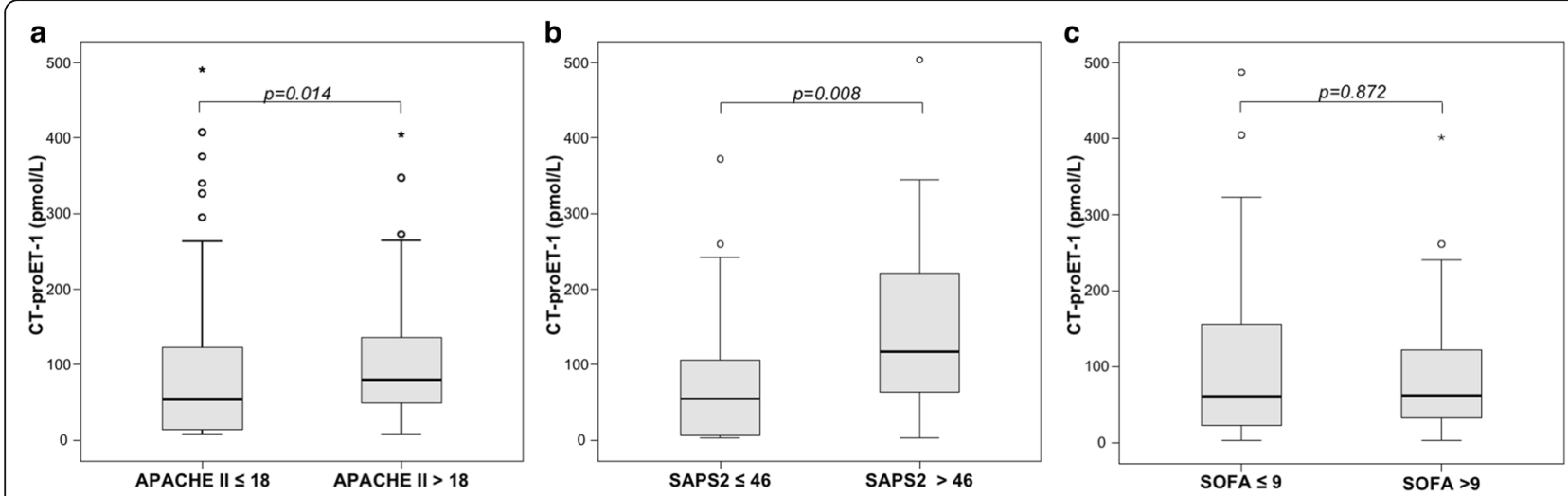

Fig. 3 Serum CT-proET-1 levels in critically ill patients are associated with disease severity. Critically ill patients with higher disease severity as represented by APACHE-II (a), SAPS 2 (b) showed significantly higher CT-proET-1 levels, while SOFA score (c) was unrelated to CT-proET-1

and hepatic dysfunction (i.e., bilirubin, prothrombin time) (Table 4). Adjusted by the factors above high CT-proET-1 $(>74 \mathrm{pmol} / \mathrm{L}$ ) remained an independent predictor of overall survival (adjusted HR 2.19, 95\%-CI 1.21-3.98).

\section{Discussion}

In this study, we demonstrate striking regulations of circulating CT-proET-1 in a large cohort of critically ill patients, supporting our hypothesis to use this stoichiometric indicator of ET-1 as a diagnostic and prognostic biomarker in intensive care medicine. We could show that CT-proET-1 is significantly elevated in critically ill patients compared to healthy controls, correlates with disease severity and organ failure, and is an independent risk predictor for ICU and overall mortality.

Previous smaller studies $[9,14,15]$ had reported an association of CT-proET-1 with short-term mortality in patients with sepsis. In contrast, one study on 99 sepsis patients that measured CT-proET-1 within $48 \mathrm{~h}$ after admission did not reproduce the relation between CTproET-1 and mortality risk [27]. Very recently, Lundberg and colleagues reported that elevated CT-proET-1 levels at ICU admission were associated with 7-day and 28-day mortality in 53 patients with septic shock [14]. Our study now extends these prior findings to a more heterogeneous, larger prospective cohort of medical ICU patients demonstrating a clear prognostic value of circulating CT-
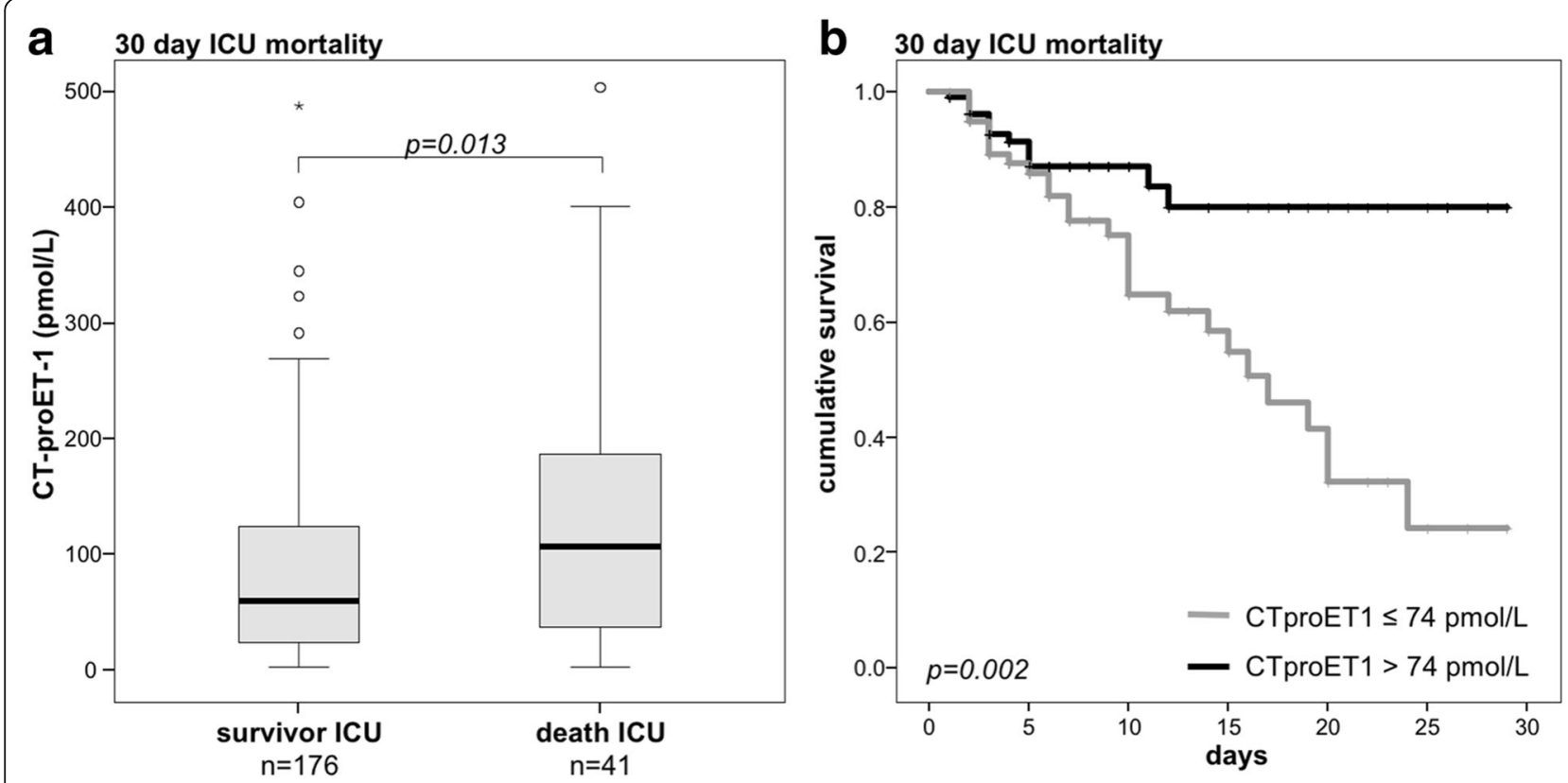

Fig. 4 Prediction of ICU mortality by CT-proET-1 serum levels. a Patients that died during the course of ICU treatment had significantly higher serum CT-proET-1 levels on admittance to ICU ( $p<0.001$; $U$ test) than survivors. b Kaplan-Meier survival curves of ICU patients are displayed for the 30-day mortality, showing that patients with $C T$-proET-1 below a cut-off value of $74 \mathrm{pmol} / \mathrm{L}$ had a better outcome at the ICU $(p=0.002$; log-rank test) 
Table 3 Uni- and multivariate Cox regression analyses for CT-proET-1 levels at ICU admission to predict ICU mortality

\begin{tabular}{|c|c|c|c|c|}
\hline & Unadjusted HR (95\%-Cl) & $p$ & Adjusted HR (95\%-Cl) & $p$ \\
\hline CT-proET-1 > $74 \mathrm{pmol} / \mathrm{L}$ & $2.658(1.375-5.137)$ & 0.004 & $2.663(1.296-5.470)$ & 0.008 \\
\hline Creatinine (per mg/dL) & - & n.s. & & \\
\hline CRP (per mg/L) & - & n.s. & & \\
\hline Bilirubin (per mg/dL) & $1.129(1.021-1.249)$ & 0.018 & - & n.s. \\
\hline Prothrombin time (per \%) & $0.988(0.977-1)$ & 0.045 & - & n.s. \\
\hline Lactate (per mmol/L) & $1.115(1.03-1.207)$ & 0.007 & $1.20(1.109-1.298)$ & $<0.001$ \\
\hline Age (per year) & $1.034(1.011-1.058)$ & 0.003 & $1.045(1.019-1.71)$ & 0.003 \\
\hline
\end{tabular}

Variables with an univariate $p<0.25$ were included in the multivariate model

Abbreviations: 95\%-Cl, 95\% confidence interval, CRP C-reactive protein, CT-proET-1 C-terminal proendothelin-1, n.s. not significant

proET-1. While patients with sepsis had higher CTproET-1 levels than ICU patients with non-septic disease, CT-proET-1 at ICU admission predicted 30-day mortality for the total patient cohort as well as for sepsis or nonsepsis patients. Moreover, we found that CT-proET-1 levels at ICU admission were even indicative of the longterm mortality risk, based on follow-up observations of about 3 years. This effect of CT-proET-1 on ICU and overall mortality was independent from single markers of organ failure or inflammation, indicating that CT-proET-1 could be useful in clinical algorithms or scores aiming at identifying high-risk patients upon ICU admission.

A prior study by Druml et al. found an association between ET-1 and the presence of an ARDS [26]. We did not observe such an association, but our study population was considerably different. While our cohort included a rather heterogeneous population of medical ICU patients with and without sepsis, Druml et al. specifically investigated ARDS patients in comparison to healthy controls, presumably with fewer confounding comorbidities than in our patient population.

Moreover, our findings might also provide important insights into the role of ET-1 (measured by CT-proET-1) in the pathogenesis of critical illness. The serum levels of CT-proET1 correlated with organ dysfunction and disease severity, but not with lactate, a marker of shock and circulatory failure. Thus, CT-proET1 reflects not a just mere hypoperfusion of tissues due to circulatory failure, but a more complex endothelial activation or dysfunction related to organ failure.

Nonetheless, our exploratory study has several limitations. These include the single center setting with the retrospective assessment of CT-proET1 in a prospectively enrolled study cohort. Moreover, we do not have

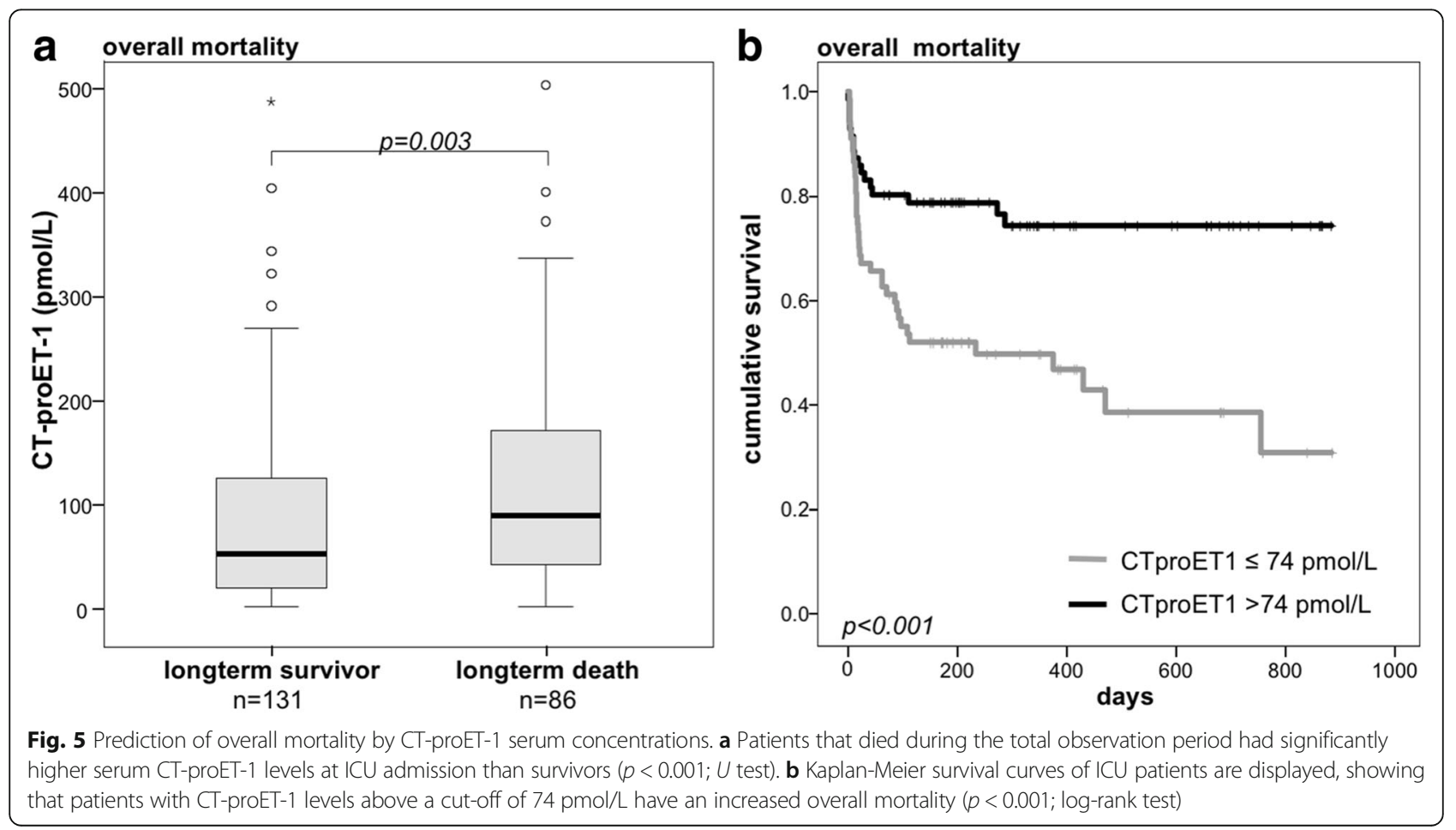


Table 4 Uni- and multivariate Cox regression analyses for CT-proET-1 levels at ICU admission to predict overall mortality

\begin{tabular}{lllll}
\hline & Unadjusted HR (95\%-Cl) & $p$ & Adjusted HR (95\%-Cl) \\
\hline CT-proET-1 > 74 pmol/L & $2.731(1.535-4.858)$ & 0.001 & $2.193(1.209-3.975)$ & - \\
Creatinine (per mg/dL) & $1.034(0.946-1.129)$ & 0.461 & - & n.s. \\
CRP (per mg/L) & $1.002(0.999-1.006)$ & 0.129 & - & n.s. \\
Bilirubin (per mg/dL) & $1.16(0.935-1.16)$ & 0.176 & - & n.s. \\
Prothrombin time (per \%) & $0.99(0.979-1)$ & 0.052 & - & n.s. \\
Lactate (per mmol/L) & $1.082(0.949-1.234)$ & 0.239 & $1.028(1.005-1.051)$ & 0.04 \\
Age (per year) & $1.036(1.014-1.059)$ & & $<.001$ &
\end{tabular}

Variables with a univariate $p<0.25$ were included in the multivariate model

Abbreviations: 95\%-CI 95\% confidence-interval, CRP C-reactive protein, CT-proET-1 C-terminal proendothelin-1, n.s. not significant

longitudinal measurements of CT-proET1, which could potentially improve the prognostic validity of this marker. Also, organ failure assessment was solely based on laboratory parameters, but no functional tests (like echocardiography or liver biopsy).

However, the hyperactivation of ET-1 in our cohort of ICU patients and the strong association of ET-1 with organ dysfunction and mortality indicate that ET-1 might be a potential drug target in critical illness and sepsis. It is tempting to speculate that antagonizing systemic supraphysiological ET-1 levels holds therapeutic potential to improve tissue perfusion. In fact, the endothelin receptor antagonist bosentan has shown positive effects on tissue perfusion $[28,29]$ and cardiac output $[30,31]$ in animal models of septic shock. Moreover, the application of bosentan in a rodent model of septic shock was even able to improve survival [32]. Interestingly, this effect was more pronounced, if bosentan was given in the hypodynamic stage after fluid resuscitation, a disease stage where specific treatment options are currently scarce. Our data corroborate to further investigate ET-1-antagonistic approaches in the ICU setting, in order to define the efficacy as well as optimal dose and timing for such an intervention.

\section{Conclusions}

Our study shows that CT-proET-1 is elevated in critically ill patients and in sepsis. It is associated with organ dysfunction and poses an independent risk factor for ICU and overall mortality. The potential as a drug treatment target in critically ill patients requires further investigations.

\section{Abbreviations}

95\%-Cl: 95\% confidence interval; APACHE: Acute Physiology and Chronic Health Evaluation; ARDS: Acute respiratory distress syndrome; AUC: Area under the curve; CRP: C-reactive protein; CT-proET-1: C-terminal

proendothelin-1; ET-1: Endothelin-1; HR: Hazard ratio; ICU: Intensive care unit; NTproBNP: N-terminal B-type natriuretic peptide; ROC: Receiver operating characteristics; SAPS 2: Simplified acute physiology score; SOFA: Sequential Organ Failure Assessment

\section{Acknowledgements}

This work was supported by the German Research Foundation (DFG; Ta434/ 5-1 and SFB/TRR57) and the Interdisciplinary Center for Clinical Research (IZKF) Aachen.

\section{Funding}

This work was supported by the German Research Foundation (DFG Ta434/ 5-1 \& SFB/TRR57) and the Interdisciplinary Centre for Clinical Research (IZKF) within the faculty of Medicine at the RWTH Aachen University.

Availability of data and materials

Not applicable.

Authors' contributions

$L B, F T$, and AK designed the study, analyzed data, and wrote the manuscript. EY performed the laboratory measurements. $\mathrm{UH}, \mathrm{CB}$, and JB collected the data and assisted in patient recruitment. CT revised the manuscript. All authors took part in the manuscript writing and approved the final manuscript.

\section{Competing interests}

The authors declare that they have no competing interests.

Consent for publication

Not applicable.

\section{Ethics approval and consent to participate}

Written informed consent was obtained from the patient, his or her spouse or the appointed legal guardian. The study protocol was approved by the local ethics committee and conducted in accordance with the ethical standards laid down in the 1964 Declaration of Helsinki (ethics committee of the University Hospital Aachen, RWTH-University, Aachen, Germany, reference number EK 150/06)

\section{Publisher's Note}

Springer Nature remains neutral with regard to jurisdictional claims in published maps and institutional affiliations.

\section{Key messages}

CT-proET-1 is a stoichiometric surrogate of the potent endogenous vasoconstrictor endothelin-1 (ET-1).

CT-proET-1 is significantly elevated in critically ill patients $(n=217)$ at admission to the ICU compared with healthy controls $(n=65)$.

CT-proET-1 is elevated in sepsis compared to non-septic ICU patients and is associated with systemic inflammation as well as organ failure.

High CT-proET-1 is an independent risk predictor for ICU and overall mortality.

These data imply that CT-proET-1 is a promising prognostic biomarker in

critical illness and that ET-1 could be a novel drug target in the pathogenesis of sepsis and septic shock.

\section{Author details}

'Department of Medicine III, RWTH-University Hospital Aachen, Pauwelsstrasse 30, 52074 Aachen, Germany. ${ }^{2}$ Medical Care Center, Dr. Stein and Colleagues, 41061 Mönchengladbach, Germany. 
Received: 20 August 2016 Accepted: 2 March 2017

Published online: 20 March 2017

\section{References}

1. Aird WC, Rangel-Frausto M, Pittet D, Costigan M, Bone R, Balk R, et al. The role of the endothelium in severe sepsis and multiple organ dysfunction syndrome. Blood. 2003;101:3765-77.

2. Barton $M$, Yanagisawa M. Endothelin: 20 years from discovery to therapy. Can J Physiol Pharmacol. 2008;86:485-98.

3. Hynynen MM, Khalil RA. The vascular endothelin system in hypertension-recent patents and discoveries. Recent Pat Cardiovasc Drug Discov. 2006;1:95-108.

4. Sugiura $M$, Inagami $T$, Kon V. Endotoxin stimulates endothelin-release in vivo and in vitro as determined by radioimmunoassay. Biochem Biophys Res Commun. 1989;161:1220-7.

5. Yamashita J, Ogawa M, Nomura K, Matsuo S, Inada K, Yamashita S, et al. Interleukin 6 stimulates the production of immunoreactive endothelin 1 in human breast cancer cells. Cancer Res. 1993:53:464-7.

6. Yoshizumi M, Kurihara H, Sugiyama T, Takaku F, Yanagisawa M, Masaki T, et al. Hemodynamic shear stress stimulates endothelin production by cultured endothelial cells. Biochem Biophys Res Commun. 1989;161:859-64.

7. Kaddoura S, Curzen NP, Evans TW, Firth JD, Poole-Wilson PA. Tissue expression of endothelin-1 mRNA in endotoxaemia. Biochem Biophys Res Commun. 1996:218:641-7.

8. Kaszaki J, Wolfárd A, Boros M, Baranyi L, Okada H, Nagy S. Effects of antiendothelin treatment on the early hemodynamic changes in hyperdynamic endotoxemia. Acta Chir Hung. 1997;36:152-3.

9. Brauner JS, Rohde LE, Clausell N. Circulating endothelin-1 and tumor necrosis factor-a: early predictors of mortality in patients with septic shock. Intensive Care Med. 2000;26:305-13.

10. Weitzberg E, Lundberg JM, Rudehill A. Elevated plasma levels of endothelin in patients with sepsis syndrome. Circ Shock. 1991;33:222-7.

11. Pittet JF, Morel DR, Hemsen A, Gunning K, Lacroix JS, Suter PM, et al. Elevated plasma endothelin-1 concentrations are associated with the severity of illness in patients with sepsis. Ann Surg. 1991:213:261-4.

12. Fenhammar J, Andersson A, Forestier J, Weitzberg E, Sollevi A, Hjelmqvist H, et al. Endothelin receptor $A$ antagonism attenuates renal medullary blood flow impairment in endotoxemic pigs. PLoS One. 2011;6:e21534.

13. Piechota-Polańczyk A, Gorąca A. Influence of specific endothelin-1 receptor blockers on hemodynamic parameters and antioxidant status of plasma in LPS-induced endotoxemia. Pharmacol Reports. 2012;64:1434-41.

14. Lundberg OHM, Bergenzaun L, Rydén J, Rosenqvist M, Melander O, Chew MS. Adrenomedullin and endothelin-1 are associated with myocardial injury and death in septic shock patients. Crit Care. 2016;20:178.

15. Schuetz $P$, Christ-Crain M, Morgenthaler NG, Struck J, Bergmann A, Müller B. Circulating precursor levels of endothelin-1 and adrenomedullin, two endothelium-derived, counteracting substances, in sepsis. Endothelium. 2008; 14:345-51.

16 Rey C, García-Hernández I, Concha A, Martínez-Camblor P, Botrán M, Medina $A$, et al. Pro-adrenomedullin, pro-endothelin-1, procalcitonin. C-reactive protein and mortality risk in critically ill children: a prospective study. Crit Care. 2013;17:R240.

17 Papassotiriou J, Morgenthaler NG, Struck J, Alonso C, Bergmann A. Immunoluminometric assay for measurement of the C-terminal endothelin1 precursor fragment in human plasma. Clin Chem. 2006;52:1144-51.

18 Koch A, Gressner OA, Sanson E, Tacke F, Trautwein C, Van Cromphaut S, et al. Serum resistin levels in critically ill patients are associated with inflammation, organ dysfunction and metabolism and may predict survival of non-septic patients. Crit Care. 2009;13:R95.

19 Singer M, Deutschman CS, Seymour CW, Shankar-Hari M, Annane D, Bauer $M$, et al. The third international consensus definitions for sepsis and septic shock (sepsis-3). Jama. 2016;315:801-10

20 The ARDS Definition Task Force. Acute respiratory distress syndrome. J. Am. Med. Assoc. 2012;307:1

21 Koch A, Sanson E, Voigt S, Helm A, Trautwein C, Tacke F. Serum adiponectin upon admission to the intensive care unit may predict mortality in critically ill patients. J Crit Care. 2011;26:166-74.

22 Koch A, Voigt S, Sanson E, Duckers H, Horn A, Zimmermann HW, et al. Prognostic value of circulating amino-terminal pro-C-type natriuretic peptide in critically ill patients. Crit Care. 2011;15:R45.
23 Koch A, Sanson E, Helm A, Voigt S, Trautwein C, Tacke F. Regulation and prognostic relevance of serum ghrelin concentrations in critical illness and sepsis. Crit Care. 2010;14:R94.

24 DeLong ER, DeLong DM, Clarke-Pearson DL. Comparing the areas under two or more correlated receiver operating characteristic curves: a nonparametric approach. Biometrics. 1988;44:837-45.

25 Wu R, Dong W, Zhou M, Cui X, Simms HH, Wang P. Ghrelin improves tissue perfusion in severe sepsis via downregulation of endothelin-1. Cardiovasc Res. 2005;68:318-26.

26 Druml W, Steltzer H, Waldhausl W, Lenz K, Hammerle A, Vierhapper H, et al. Endothelin-1 in adult respiratory distress syndrome. Am Rev Respir Dis. 1993;148:1169-73

27 Guignant C, Venet F, Voirin N, Poitevin F, Malcus C, Bohé J, et al. Proatrial natriuretic peptide is a better predictor of 28-day mortality in septic shock patients than proendothelin-1. Clin Chem Lab Med. 2010:48:1813-20.

28 Krejci V, Hiltebrand LB, Erni D, Sigurdsson GH. Endothelin receptor antagonist bosentan improves microcirculatory blood flow in splanchnic organs in septic shock. Crit Care Med. 2003;31:203-10.

29 Oldner A, Wanecek M, Goiny M, Weitzberg E, Rudehill A, Alving K, et al. The endothelin receptor antagonist bosentan restores gut oxygen delivery and reverses intestinal mucosal acidosis in porcine endotoxin shock. Gut. 1998; 42:696-702

30 Wanecek M, Oldner A, Rudehill A, Sollevi A, Alving K, Weitzberg E. Cardiopulmonary dysfunction during porcine endotoxin shock is effectively counteracted by the endothelin receptor antagonist bosentan. Shock. 1997; 7:364-70.

31 Weitzberg E, Hemsén A, Rudehill A, Modin A, Wanecek M, Lundberg JM. Bosentan-improved cardiopulmonary vascular performance and increased plasma levels of endothelin-1 in porcine endotoxin shock. Br J Pharmacol. 1996;118:617-26.

32 Iskit AB, Senel I, Sokmensuer C, Guc MO. Endothelin receptor antagonist bosentan improves survival in a murine caecal ligation and puncture mode of septic shock. Eur J Pharmacol. 2004;506:83-8.

\section{Submit your next manuscript to BioMed Central and we will help you at every step:}

- We accept pre-submission inquiries

- Our selector tool helps you to find the most relevant journal

- We provide round the clock customer support

- Convenient online submission

- Thorough peer review

- Inclusion in PubMed and all major indexing services

- Maximum visibility for your research

Submit your manuscript at www.biomedcentral.com/submit
C) Biomed Central 